\title{
A Doppler statistic for zero autocorrelation waveforms
}

\author{
John J. Benedetto, Jeffrey Donatelli, Ioannis Konstantinidis, and Christopher Shaw \\ Norbert Wiener Center \\ Department of Mathematics \\ University of Maryland \\ College Park MD 20742
}

\begin{abstract}
There are several natural constructions of constant amplitude zero autocorrelation (off the DC-component) waveforms. We adopt a construction for waveforms of length $K$, where $K$ is a non-square-free integer. This property of $K$ is used in the derivation of a frequency shifting (Doppler) detection algorithm which we derive. There are number theoretic propeties which account for the properties of this algorithm, as well as for analogous but different properties in the square-free case. A variety of relevant examples is given along with the technical rationale for the algorithm.
\end{abstract}

\section{INTRODUCTION}

We shall analyze a certain class of unimodular low correlation waveforms. In fact, the specific waveforms with which we deal are of finite length and have 0 -autocorrelation off of the DC-component. Such waveforms are referred to as CAZAC waveforms, viz., Constant Amplitude Zero Autocorrelation. There is an extensive literature on CAZACs because of the importance of such waveforms in communications and coding theory, e.g., [1], [2], [3], [4], [5], [6], [7], [8], [9]. Our own interest is based on the importance of waveform design in several aspects of modern radar [10], [11], [12] .

We begin in Section II-A by defining the autocorrelation and crosscorrelation of signals of finite length $K$, as well as the ambiguity function of a given waveform of finite length. It is noted that the usual notion of the ambiguity function on the real line $\mathbb{R}$ is the analogue of our discrete definition. In Section II-B properties of CAZACs are recorded including the important fact that $u$ is CAZAC if and only if its discrete Fourier transform $U$ is CAZAC. Some of the CAZACs we have used, and whose software [13] we have made available, have the property that $K$ is not square-free, and we give a typical example.

We recall some RADAR fundamentals in Section III that we require for our setting and statistic. In particular, we describe the role of constant amplitude in Section III-A zero autocorrelation in Section $\amalg$ II-B and the modulation scheme to which our CAZACs will be applied in Section III-C. Section IV deals with a pristine form of the Doppler effect, and we construct a statistic in order to compute frequency shifting and, therefore, target speed. We state a fundamental theoretical result as well as examples, and conclude in Section V

\section{CAZAC}

\section{A. Correlations}

Let $e_{m}(n)=e^{\frac{-2 \pi i m n}{K}}$ and $\mathbb{Z}_{K}=\{0,1, \ldots, K-1\}$. Let $u, v: \mathbb{Z}_{K} \rightarrow \mathbb{C}$ be (discrete) $K$-periodic waveforms. The crosscorrelation of $u$ and $v$ is

$$
C_{u, v}(m)=\frac{1}{K} \sum_{k=0}^{K-1} u(m+k) \bar{v}(k)
$$

for $m=0,1, \ldots, K-1$.

The autocorrelation of $u$ is its crosscorelation with itself: $A_{u}(m)=C_{u, u}(m)$.

The (discrete) ambiguity function of $u, A: \mathbb{Z}_{K} \times \mathbb{Z}_{K} \rightarrow \mathbb{C}$, is defined as

$$
A_{u}(j, k)=C_{u, u e_{k}}(j)=\frac{1}{K} \sum_{m=0}^{K-1} u(m+j) \overline{u(m)} e^{\frac{2 \pi i m k}{K}} .
$$

It is natural to refer to $A$ as the ambiguity function of $u$, since in the usual setting on the real line $\mathbb{R}$, the analogue ambiguity function is

$$
\begin{aligned}
A_{u}(t, \gamma) & =\int_{\widehat{\mathbb{R}}} U\left(\omega-\frac{\gamma}{2}\right) \overline{U\left(\omega+\frac{\gamma}{2}\right)} e^{2 \pi i t\left(\omega+\frac{\gamma}{2}\right)} d \omega \\
& =\int_{\mathbb{R}} u(s+t) \overline{u(s)} e^{2 \pi i s \gamma} d s,
\end{aligned}
$$

where $U$ is the Fourier transform of $u: \mathbb{R} \rightarrow \mathbb{C}, \widehat{\mathbb{R}}$ is $\mathbb{R}$ considered as the spectral domain, and the $L^{2}$ norm (finite energy) of $u$ is designated by $\|u\|_{2}$.

A $K$-periodic waveform $u$ is a constant amplitude zero autocorrelation waveform (CAZAC) if $|u(m)|=1, m=$ $0,1, \ldots, K-1$, and its autocorrelation $A_{u}(m)$ is 0 for $m=$ $1, \ldots, K-1$.

\section{B. Properties}

We list a few interesting properties of (discrete) $K$-periodic waveforms $u: \mathbb{Z}_{K} \rightarrow \mathbb{C}$.

- If $u$ has zero autocorrelation off the DC-component (ZAC), then it is of full bandwidth. Moreover, its Fourier transform $\hat{u}$ has constant magnitude.

- A waveform $u$ has constant amplitude (CA) if and only if the discrete Fourier Transform (DFT) of $u$ has the ZAC property. 
- $u$ is CAZAC if and only if its DFT is also CAZAC.

Generally, there are distinct CAZAC waveforms of any given length $K$, and different constructions of CAZACs may yield different applicability. Figure 1 provides an example of a CAZAC with length 75. In particular, CAZAC waveforms are broadband, and the best finite energy approximants by waveforms of smaller bandwidth are products of $\hat{u}$ by characteristic functions. The relation of these properties to RADAR is explained in Section III.

\section{RADAR FUNDAMENTALS}

The principle underlying the object detection method with pulsed RADAR is quite elementary - it asserts that detecting a reflection of an electromagnetic wave pulse signifies the presence of an obstacle in the wave's propagation path which caused the reflection. Since the speed of the wave pulse is constant and equal to the speed of light $c$, the time delay between the original pulse transmission and the detection of its reflection by the RADAR antenna is equal to the time it takes a light particle to travel twice the distance between antenna and target. Thus, the distance to the target can be estimated to the extent that this time delay can accurately and unambiguously be measured.

In the following, we shall recall some of the issues associated with this measurement, in order to motivate the need for sophisticated waveform design in RADAR. We shall also discuss the Doppler effect, and additional constraints and trade-offs imposed by the need for the estimation of a target's speed relative to the source.

\section{A. Constant Amplitude}

The simplest form of pulsed RADAR can be described as follows: An RF wave of frequency $f$ is transmitted for a time period $\tau_{1}$, after which the antenna monitors for its return for another time interval $\tau_{2}$. The pulse repetition interval (PRI) $\tau_{1}+\tau_{2}$ represents a single cycle in the operation of the pulse RADAR. Clearly, we must have $\tau_{1}<\tau_{2}$ in order to be able to process the return completely. More importantly, $\tau_{2}$ must be long enough to allow for signals to return from all targets within the RADAR's range in the same PRI that they originated.

Another constraint on the length of $\tau_{1}$ comes from energy considerations. For the sake of simplicity, let us assume that an omnidirectional source antenna $\mathrm{A}$ is emitting an electromagnetic wave of constant power $P_{s}$ from a point located at the origin ( $s$ is for "send"). Now let us consider a stationary target $\mathrm{B}$ at a distance $R$ from the origin. Since the antenna is omnidirectional, wave energy is distributed evenly over the surface of the sphere of radius $R$, and hence the received power $P_{t}$ at the target $\mathrm{B}$ is inversely proportional to $R^{2}$ ( $t$ is for "target"). The target then acts as a secondary source with power $P_{t} \sim \frac{P_{s}}{R^{2}}$, emitting reflected waves that arrive back at the source. Thus, the power $P_{r}$ received back at the point $\mathrm{A}$ is

$$
P_{r} \sim \frac{P_{t}}{R^{2}} \sim \frac{P_{s}}{R^{4}}
$$

( $r$ is for "receive"). There are many factors that affect the constant of proportionality in this form of the RADAR equation, including the target's cross sectional area, geometry, and material reflective properties, as well as losses associated with the propagation medium. However, the crucial factor remains the inverse fourth power dependence on the emitted signal strength. This shows that range is crucially dependent on the amount of energy transmitted to illuminate the scene during $\tau_{1}$. Thus, it is also important to maximize not only the power of the transmitted wave pulse, and hence its amplitude, but also the total energy carried by it into the scene, and hence the length of $\tau_{1}$.

Therefore, for a given noise power level, constant amplitude waveforms have the advantage of extending the detection range over amplitude modulated ones. This is because constant amplitude wave pulses maximize the power received, since they can be transmitted at peak power, as opposed to variable amplitude ones. At the same time, the total energy transmitted is the integral of power over time. Thus, all other things being equal, longer wave pulses carry more energy into a scene. Hence, they allow returns from farther away to remain clearly above the noise power levels.

\section{B. Matched filtering and the ambiguity function}

The disadvantage of using simple pulses comes from the constraints they introduce in range resolution. Let us assume that the pulse transmitted is given by the function $s(t)=$ $\mathbf{1}_{\left[0, \tau_{1}\right]}$, the characteristic function of the transmission interval. Further assume that the reflection comes from a single point target, located at a distance $d$. Then the return signal has the form

$$
r(t)=a s(t-T),
$$

where the delay $T$ is related to the distance $d$ by the speed of light $c$ via $c=\frac{2 d}{T}$, and $a>0$ is an attenuation factor. It can be shown that

$$
C_{r, s}(T)=\sup _{t}\left|C_{r, s}(t)\right|,
$$

where $C_{r, s}$ is $L^{2}(\mathbb{R})$ crosscorrelation, and the maximum system response is given by the matched filter $\widehat{s^{\star}}(\gamma)=$ $\overline{a \hat{s}(\gamma)} e^{-2 \pi i T \gamma}$.

In the setting we have discussed so far, $s$ is the characteristic function of the transmission interval, so $\left|C_{r, s}(t)\right|$ is a tent function centered at $T$. In the presence of additive Gaussian white noise, while the matched filter $\widehat{s^{\star}}$ still achieves the maximum signal-to-noise ratio, $\sup _{t}\left|C_{r, s}(t)\right|$ is no longer guaranteed to be exactly at $T$. Thus, $T$ can only be determined to be in the range where $\left|C_{r, s}(t)\right|$ remains above the noise threshold, and this is an interval around $T$ whose length is proportional to $\tau_{1}$.

Thus, the shape of the graph of $C_{r, s}(t)$ determines the precision with which an estimate of the time of return can be measured, and this is directly proportional to the precision of the measurement of the target's distance. Equivalently, one considers the autocorrelation $A_{s}(t)$. The types of signals whose autocorrelation decays rapidly away from the DC 
component and remains small away from it provide the best localization.

What is remarkable about CAZAC sequences is that they provide explicit examples where the autocorrelation is precisely equal to zero away from the DC component. This means that, in the discrete case, we can achieve the best localization possible. In the next subsection, we explain how to encode these signals using quadrature amplitude modulation.

\section{Quadrature amplitude modulation and demodulation}

In general, the transmitted pulse consists of a signal $u$ : $\mathbb{R} \rightarrow \mathbb{C}$ modulated by an RF wave $w(t)=\exp (2 \pi i f t)$, of constant frequency $f$, called the carrier wave. Notice that the complex exponential form of the carrier wave refers to its full electromagnetic behavior; it is a cosine of frequency $f$ in terms of electric current. Here, we assume that the function $u(t)$ is supported on the interval $\tau_{1}$, and write

$$
\begin{aligned}
u(t) & =A(t) \exp (2 \pi i \theta(t)) \\
& =A(t) \cos (2 \pi \theta(t))+i A(t) \sin (2 \pi \theta(t)),
\end{aligned}
$$

where non-negative $A: \mathbb{R} \rightarrow \mathbb{R}$ is the signal amplitude and $\theta: \mathbb{R} \rightarrow \mathbb{T}$ is its phase. The real part

$$
I=A(t) \cos (2 \pi \theta(t))
$$

of $u$ is called the in-phase component, and the imaginary part

$$
Q=A(t) \sin (2 \pi \theta(t))=A(t) \cos \left(2 \pi \theta(t)+\frac{\pi}{2}\right)
$$

is the quadrature component. These components are multiplied with two RF waves that are in quadrature phase, namely $\cos (2 \pi f t)$ and $\sin (2 \pi f t)$, and then summed to produce the modulated signal

$$
\begin{aligned}
s(t)= & A(t) \cos (2 \pi \theta(t)) \cos (2 \pi f t) \\
& +A(t) \sin (2 \pi \theta(t)) \sin (2 \pi f t) \\
= & A(t) \cos \{2 \pi i(f t-\theta(t))\} .
\end{aligned}
$$

Again, the full electromagnetic model for the transmitted wave is

$$
s(t)=\exp \{2 \pi i(f t-\theta(t)\} .
$$

Hence, the phase information of $u$ is encoded in the transmitted signal as a phase delay. Assuming that $\theta$ is differentiable, we see that the instantaneous frequency of the modulated wave is now $f+\theta^{\prime}$, and its amplitude is $A$. Since constant amplitude pulses are preferable, we shall limit our discussion to unitary signals $u(A=1)$.

Quadrature modulated signals can be demodulated using a parallel process for recovering the $\mathrm{I}$ and $\mathrm{Q}$ components separately and then adding them together. First, the original inphase quadrature component $\cos (2 \pi i f t)$ is used to modulate the received signal

$$
r(t)=r(t) \cos (2 \pi i f t)=\cos (2 \pi i f t) \cos \{2 \pi i(f t-\theta(t))\},
$$

and then the resulting wave is filtered with an appropriate sinc function to cut off the harmonics above $f$. A similar process (using $\sin (2 \pi i f t)$ ) is applied to recover the quadrature component. Thus, the full original signal $u$ is recovered.

In digital systems, modulation and subsequent demodulation are performed at regularly sampled time intervals. Typically, the carrier wave frequency is in the order of $\mathrm{GHz}$, and the sampling rates are in the order of $\mathrm{MHz}$. This implies that $\theta$ becomes a step function

$$
\theta(t)=\sum_{m} \theta[m] \mathbf{1}_{I_{m}}(t)
$$

where the step intervals $I_{m}=\left[0, \frac{\tau_{1}}{K}\right]+m \frac{\tau_{1}}{K}$ have a size of approximately 1000 carrier cycles.

\section{DOPPLER STATISTIC}

\section{A. Doppler effect}

The most basic form of the Doppler effect states that the wavelength of a pure sinusoidal wave as measured by a receiver varies according to the relative speed of the receiver to the source. If $\lambda_{v}$ denotes the wavelength as measured by a receiver moving towards the source at a constant speed $v$, then

$$
\frac{\lambda_{0}}{\lambda_{v}}=\frac{c+v}{c} .
$$

This is justified if one considers that the time it takes for a moving receiver to traverse a full wavelength is shortened by its speed relative to the traveling wave (as opposed to the stationary receiver). Equivalently,

$$
\frac{f_{v}-f_{0}}{f_{0}}=\frac{v}{c},
$$

where $f_{v}=\frac{1}{\lambda_{v}}$ is the measured frequency. The same reasoning carries through when one considers moving sources and stationary targets, as only relative speed matters. Thus, in the case of RADAR, when a signal is reflected off of a moving target, we expect to see its frequency shifted by

$$
f_{D}=2 \frac{v}{c} f
$$

We are going to focus on a single return, from a single point target, located at a distance $d$ and traveling at a speed $v$ along the line of sight of the radar. In this idealized situation, the received signal can be modeled as

$$
r(t)=s(t-T) \exp \left(-2 \pi i f_{D}(t-T)\right),
$$

where $T=\frac{2 d}{c}$ is the time delay, and $f_{D}=2 \frac{v}{c} f$ is the frequency shift due to the Doppler effect.

\section{B. CAZAC statistic}

In the digital case, we are led to examine the properties of the discrete ambiguity function $A_{u}(j, k)=C_{u, u e_{k}}(j)$, where $j$ corresponds to the time delay and $k$ corresponds to the Doppler shift.

There is a fundamental Doppler tolerance problem: construct a statistic to determine an unknown Doppler frequency shift. In order to address this problem we have the following result which is valid for certain subclasses of non-square-free integers [14]. 
Theorem 1: Let $K=M \times N \times N$ and let $k=0,1, \ldots, K-$ 1. The quantity $\left|C_{u, u e_{k}}(\cdot)\right|$ is $N$-periodic as a function of $k$, i.e., there are at most $N$ different graphs of $\left|C_{u, u e_{k}}(\cdot)\right|$. Also, given $k,\left|C_{u, u e_{k}}(j)\right|=0$ for all $j \neq(-k) \bmod M N$. Further, $\sum_{j=0}^{K-1}\left|C_{u, u e_{k}}(j)\right|^{2}=1$.

This theorem translates to the fact that the discrete ambiguity function $A_{u}$ is mostly supported on a single point when restricted to lines corresponding to constant $k$, where $k$ is a given Doppler shift. Hence, range resolution remains optimal in the presence of Doppler effects. It also follows from the theorem that the values at the sidelobes remain below a fixed threshold, depending on $K$ alone (Fig. 2).

There are different CAZAC constructions that lead to different peak sidelobe values (Fig. 3). Figure 3(c) shows a CAZAC $u$ for which the support of $A_{u}$ is a single point when restricted to lines corresponding to constant $k$ ( $k$ a given Doppler shift), and similarly for constant $j$ ( $j$ a given time shift). This flexibility allows us to construct a library of CAZAC waveforms for scheduling purposes.

One particular application is the case of using a conjugate pair of CAZACs, $u$ and $\bar{u}$. Note that the ambiguity function of $\bar{u}$ satisfies

$$
\left|A_{\bar{u}}(j, k)\right|=\left|A_{u}(-j, k)\right| .
$$

Hence, the function $\left|A_{\bar{u}}\right|\left|A_{u}\right|(j, k)$ is localized at a single point in the time-frequency plane.

\section{CONCLUSION}

We have presented results on a certain class of unimodular low correlation waveforms, called CAZAC, viz., Constant Amplitude Zero Autocorrelation (off the DC component). We provided motivation from the theory of digital pulsed RADAR, and constructed a Doppler statistic $\left|C_{u, u e_{k}}(j)\right|$ in order to compute frequency shifting and, therefore, target speed. This statistic is not only elementary to explain, but also proves useful and accurate. Note that if one graphs only $\operatorname{Re} A(j, k)=$ $\operatorname{Re} C_{u, u e_{k}}(j)$ then the statistic sometimes fails. We have stated a fundamental theoretical result in this respect, as well as provided examples to illuminate our results. Further, we point out that there are unresolved "arithmetic" complexities which are affected by waveform structure and length; and that our noise analysis is ongoing.

\section{ACKNOWLEDGEMENT}

This work was supported in part by AFOSR grant AFOSRFA9550-05-1-0443, ONR grant ONR-N000140610002, and by DARPA funding under NRL grant N00173-06-1-G006.

\section{REFERENCES}

[1] D. C. Chu, "Polyphase codes with good periodic correlation properties," IEEE Trans. Inform. Theory, vol. 18, pp. 531-532, 1972.

[2] R. L. Frank and S. A. Zadoff, "Phase shift pulse codes with good periodic correlation properties," IEEE Trans. Inform. Theory, vol. 8, pp. 381-382, 1962.

[3] T. Helleseth and P. V. Kumar, Sequences with low correlation. Elsevier Publishers, 1998, ch. 21.

[4] A. Milewski, "Periodic sequences with optimal properties for channel estimation and fast start-up equalization," IBM J. Res. Dev., vol. 27, pp. 426-430, 1983.

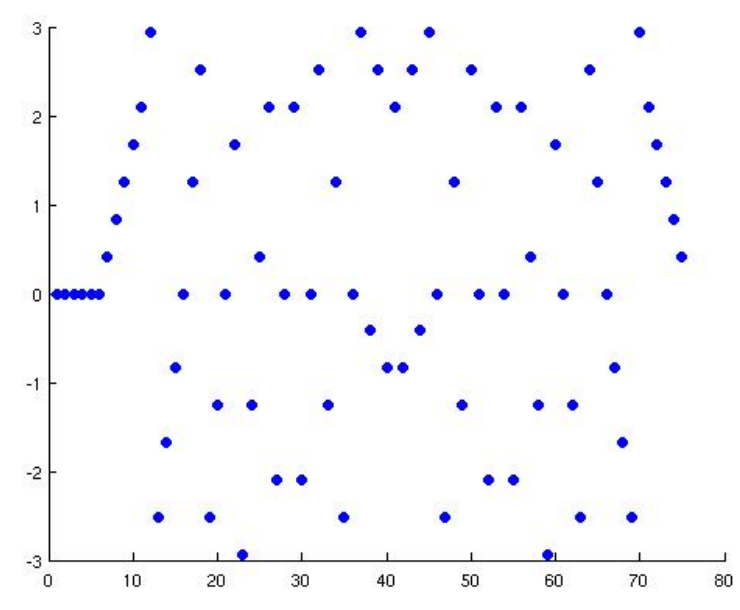

Fig. 1. A discrete CAZAC signal of length 75 ; the graph plots the phase in radians.

[5] U. H. Rohrs and L. P. Linde, "Some unique properties and applications of perfect squares minimum phase cazac sequences," in Proc. South African Symp. on Communications and Signal Processing, 1992, pp. $155-160$.

[6] R. Turyn, Sequences with small correlations. New York: John Wiley \& Sons, Inc., 1968, pp. 195-228.

[7] J. Ng, K. Letaief, and R. Murch, "Complex optimal sequences with constant magnitude for fast channel estimation initialization," IEEE Transactions on Communications, vol. 46, no. 3, pp. 305-308, 1998.

[8] E. Gabidulin and V. Shorin, "Unimodular perfect sequences of length $p^{s}$, IEEE Transactions on Information Theory, vol. 51, no. 3, pp. $1163-$ $1166,2005$.

[9] W. H. Mow, "A new unified construction of perfect root-of-unity sequences," in IEEE 4th International Symposium on Spread Spectrum Techniques and Applications, vol. 3, Sep 1996, pp. 955-959.

[10] J. R. Klauder, "The design of radar signals having both high range resolution and high velocity resolution," Bell System Technical Journal, vol. 39, pp. 809-820, 1960.

[11] J. R. Klauder, A. C. Price, S. Darlington, and W. J. Albersheim, "The theory and design of chirp radars," Bell System Technical Journal, vol. 39, pp. 745-808, 1960.

[12] N. Levanon and E. Mozeson, Radar Signals. Wiley, 2004.

[13] J. J. Benedetto and J. F. Ryan, "Software package for CAZAC code generators and Doppler shift analysis," 2004, www.math.umd.edu/ jjb/ cazac

[14] J. J. Benedetto and J. Donatelli, "A doppler statistic associated with low correlation waveforms of finite length." 


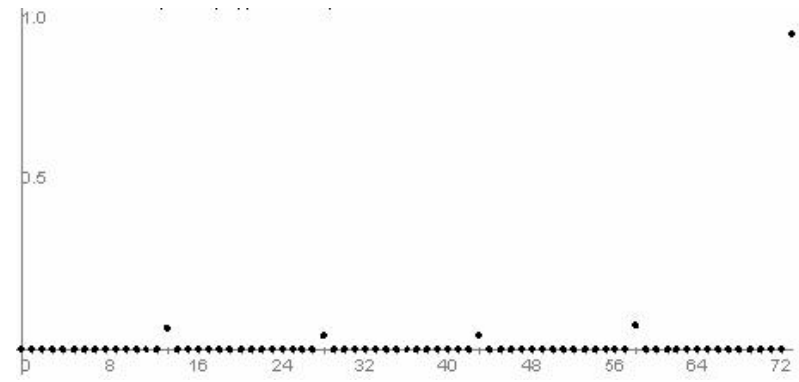

(a)

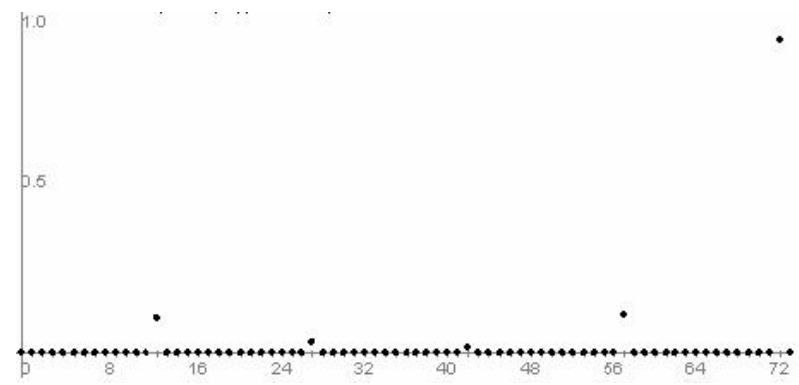

(b)

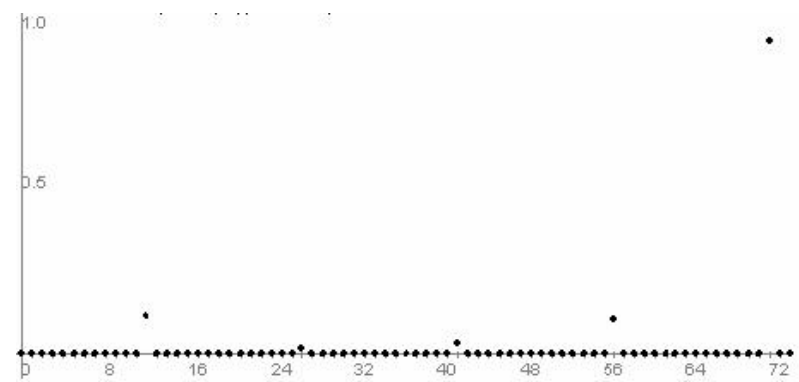

(c)

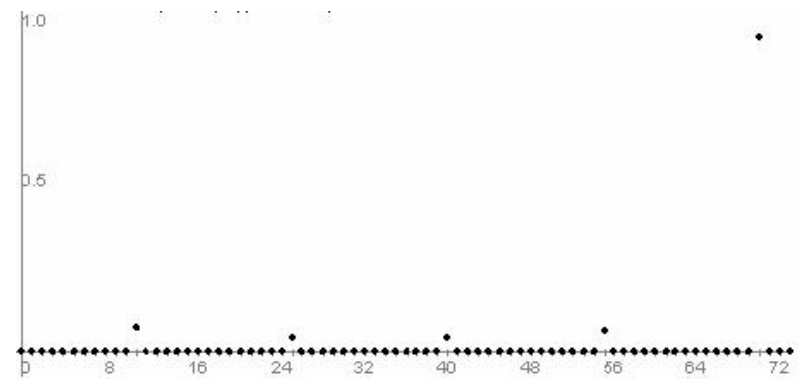

(d)

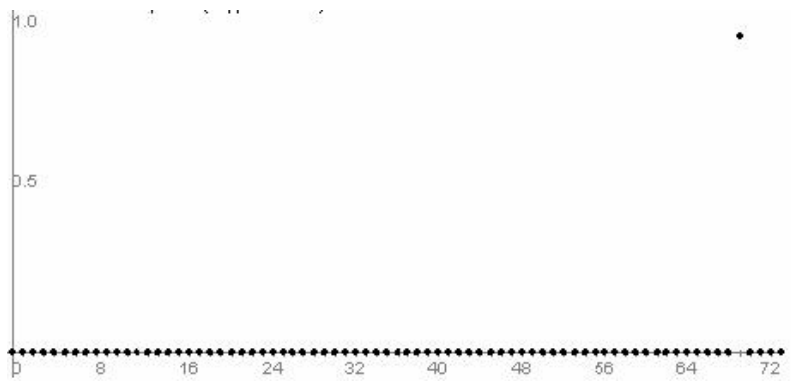

(e)

Fig. 2. Doppler statistic for the CAZAC in Fig. 1. (a) - (e) graphs of $\left|C_{u, u e_{k}}(\cdot)\right|$ for $k=1, \ldots, 5$, respectively.

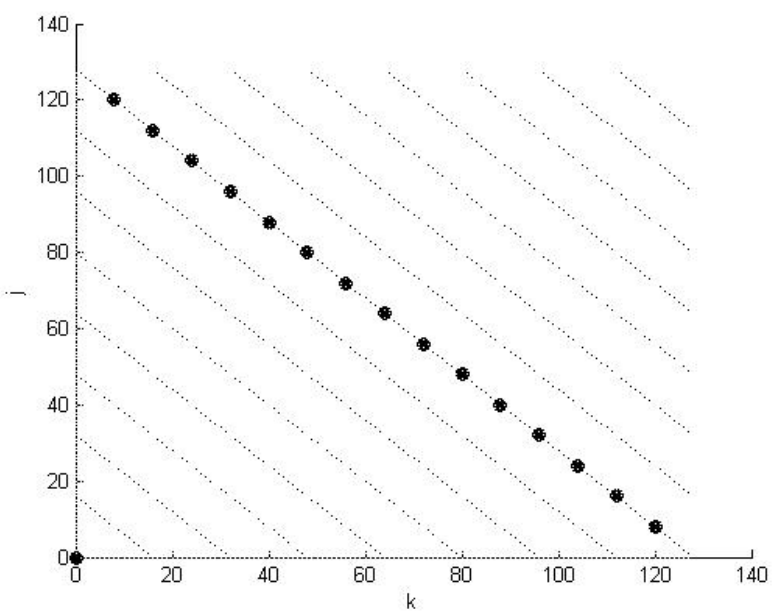

(a)

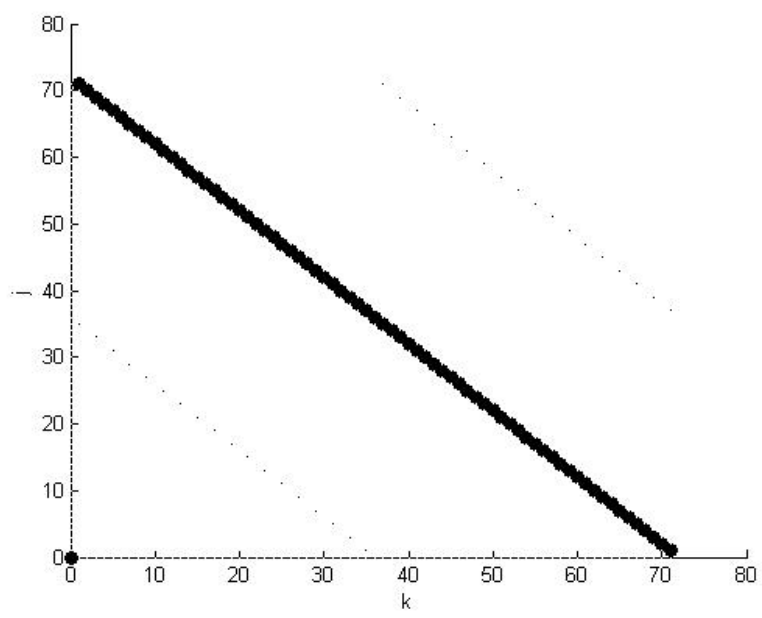

(b)

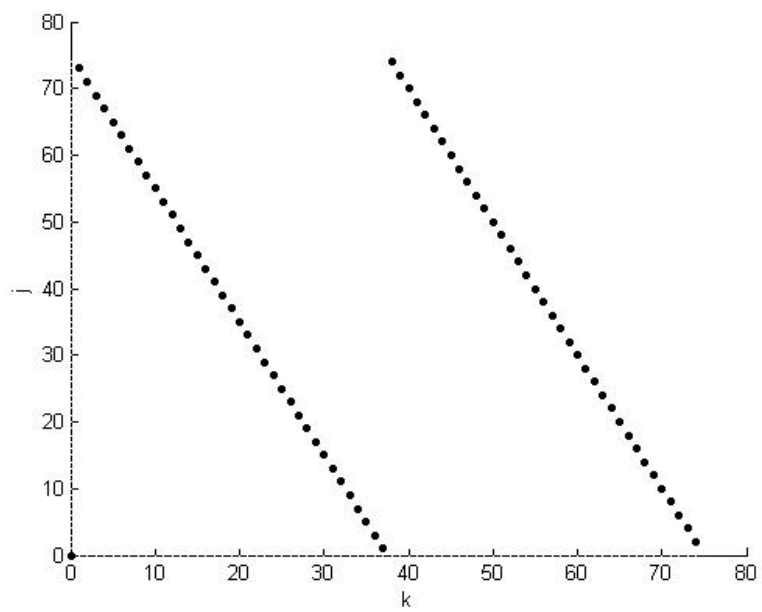

(c)

Fig. 3. Scatter plot of the discrete ambiguity function $A_{u}(k, j)$ in the $(k, j)$ time-frequency plane; points have radii proportional to the value of $\left|A_{u}(k, j)\right|$. (a) - (c): $u$ are CAZAC sequences of lengths 128,72 , and 75, respectively. 